\title{
Theoretical interpretation of the toroidal rotation velocity observed in Alcator C-Mod Ohmic H-mode discharges
}

\author{
A.L. Rogister, J.E. Rice ${ }^{1}$, A. Nicolai, A. Ince-Cushman ${ }^{1}$, \\ S. Gangadhara ${ }^{1}$ and Alcator C-Mod Group ${ }^{1}$ \\ Institut für Plasmaphysik, Forschungszentrum Jülich GmbH, EURATOM Association, \\ Trilateral Euregio Cluster, D-52425 Jülich, Germany \\ ${ }^{1}$ Plasma Science and Fusion Center, Massachusetts Institute of Technology, Cambridge, \\ MA, USA \\ E-mail: a.rogister@fz-juelich.de
}

Received 15 March 2002, accepted for publication 24 June 2002

Published 20 August 2002

Online at stacks.iop.org/NF/42/1144

\begin{abstract}
It is shown that neoclassical theory explains quite well the origin of the co-current toroidal rotation velocity measured in the core of stationary Alcator C-Mod edge localized mode (ELM)-free Ohmic high confinement (H)-mode discharges. Both edge and core toroidal rotation velocity profiles are determined to a good approximation by the edge ion temperature and density pedestals, where the gradients are large and the plasma is in the high collisionality regime. Under these conditions, the predicted radial electric field profile is similar to those measured in the DIII-D tokamak whereas the usual expression for the poloidal velocity is modified by finite Larmor radius (FLR) effects. Over the entire plasma cross section, the expression of the toroidal velocity can approximately be cast as the product of a dimensionless non-local functional of the pedestal normalized profiles $T_{\mathrm{i}}(r) / T_{\mathrm{i}}\left(r_{\mathrm{inf}}\right)$ and $N_{\mathrm{i}}(r) / N_{\mathrm{i}}\left(r_{\mathrm{inf}}\right)$ with powers of the plasma density, temperature, safety factor and magnetic field at the pedestal inflexion point $r_{\text {inf }}$ provided the FLR related corrections are independent of the latter parameters. The collapse of the core toroidal rotation velocity when either an internal transport barrier forms (that leads to impurity accumulation), or the plasma experiences a transition from the $\mathrm{H}$ - to the low confinement (L)-mode, or ELMs appear, and the spin up at the $\mathrm{L}-\mathrm{H}$ transition are also explained. In the edge region, power balance is consistent with the prediction from subneoclassical ion energy transport theory at high collisionality. The role of charge exchange neutrals is discussed and the critical density above which they are expected to noticeably slow down the rotation is estimated. The toroidal velocity gradient predicted by theory at the edge of the ELM-free Ohmic H-mode discharge mainly under study $\left(q_{\mathrm{s}}=3.4\right)$ is near the onset value for the Kelvin-Helmholtz $(\mathrm{K}-\mathrm{H})$ parallel velocity shear (PVS) instability; this result is very interesting since a transition from ELM-free to enhanced $\mathrm{D}_{\alpha}$ (EDA) H-modes occurs at $q \cong 3.5-4$; the PVS K-H instability appears to have the characteristics of the 'quasi-coherent' mode that is present in all EDA plasmas, but not in ELM-free H-modes.
\end{abstract}

PACS numbers: 52.55

\section{Introduction}

In a liquid in contact with a heat source, the temperature gradient generates ascending Benard cells which transfer the energy to the surface in contact with the cooling atmosphere. Similarly, in a magnetically confined plasma, the inherent temperature and density gradients are free energy sources which generate micro-instabilities resulting in propagating turbulence and anomalous energy transport to the edge. Anomalous transport has plagued the performance of tokamaks since they went into operation. The discovery of the high confinement $(\mathrm{H})$-mode [1] was thus a major step in fusion research. This regime is characterized by temperature and density pedestals which, usually, about double the energy content of the discharge. H-mode plasmas are almost exclusively observed in tokamaks with axisymmetric magnetic divertors; moreover, the transition from the low confinement (L)-mode to the $\mathrm{H}$-mode requires a minimum power input and thus a minimum energy flux through the edge. This constraint explains why the $\mathrm{H}$-mode has first been obtained in discharges with auxiliary heating.

It has long been recognized that radial electric fields and, therefore, plasma rotation play an important role in the L- to H-mode transition [2-7]. External momentum input and 
torques are associated with neutral beam injection heating, but negligibly so with ion cyclotron radio frequency (ICRF) wave heating [8]. Realization of the H-mode in ICRF-heated plasmas [9] thus rules out that momentum input should be necessary to trigger the transition. It has been argued that ICRF waves can give rise to an inward shift of energetic ion orbits and, therefore, to charge separation, a radial electric field and $\boldsymbol{E} \times \boldsymbol{B}$ rotation [10]. Realization of the H-mode in high-power density Ohmic Alcator C-Mod plasmas [11], however, shows that plasma rotation can develop spontaneously: the toroidal rotation velocity of impurities measured on the magnetic axis is actually observed to jump from a negligible negative to a large positive $\left(\sim 35 \mathrm{~km} \mathrm{~s}^{-1}\right)$ value at the transition (the toroidal axis is oriented in the direction of the plasma current). Explaining that intrinsic toroidal rotation may shed light on the mechanism of the L- to H-mode transition by confirming some hypothesis or denying others.

We show in this paper that the neoclassical equations which were derived in [12] for the toroidal and the poloidal rotation velocities in high collisionality plasmas explain quite accurately the experimental results in edge localized mode (ELM)-free Ohmic H-mode discharges [11]. Moreover, the sub-neoclassical heat flux predicted in $[6,7]$ is quite close to the heat flux calculated from power balance at the plasma edge and the profile of the radial electric field obtained from the radial momentum balance equation is similar to those measured in DIII-D [13,3].

The theory [12] predicts that the toroidal velocity at a position $r$ inside the plasma is proportional to the integral from the last closed flux surface (LCFS) up to $r$ of the product $U_{\theta, \mathrm{i}} \partial_{r} \ln T_{\mathrm{i}}$, i.e. to $\Sigma_{\mathrm{d}}\left[\left(T_{\mathrm{i}}^{\prime}\right)^{2}\left(T_{\mathrm{i}}\right)^{-1} L_{T_{\mathrm{i}}}\right]_{\mathrm{d}} \approx \Sigma_{\mathrm{d}}\left[T_{\mathrm{i}}^{\prime}\right]_{\mathrm{d}}$; here, $U_{\theta, \mathrm{i}}$ is the poloidal velocity, a factor $\left[L_{T_{\mathrm{i}}}\right]_{\mathrm{d}}$ has been introduced as the measure of the successive integration domains $d$ (edge pedestal, core) and we have assumed $U_{\theta, \mathrm{i}} \propto T_{\mathrm{i}}^{\prime} / e B_{\varphi}$ for the estimation (this expression conforms with conventional neoclassical theory [14], but is modified at the plasma edge due to the large gradients and finite Larmor radius (FLR) effects; the latter are also responsible for the reduction of the neoclassical heat flux). Plasma viscosity does not enter the equations if the system is stationary, neutrals play a negligible role, and there is no momentum source. In H-mode plasmas, $T_{\mathrm{i}}^{\prime}$ is much larger in the pedestal region than in the core; the contribution from the latter to the above integral is thus negligible. As a result, the toroidal rotation velocity measured in the plasma centre is determined by the temperature gradient in the pedestal if the relation between the toroidal and poloidal velocities obtained in the framework of the neoclassical theory for the high collisionality regime remains valid, order of magnitude wise, in the other collisionality regimes or in the presence of turbulence. (This is likely to be the case as (i) that relation is independent of the transport coefficients (it reflects, rather, the plasma anisotropy in the directions parallel and perpendicular to $\boldsymbol{B}$ ) and (ii) trapped particles do not participate in the toroidal velocity (they contribute, however, to magnetic pumping; thus the different proportionality coefficients between the poloidal velocity and the temperature gradient in the different collisionality regimes; they may similarly affect the toroidal velocity if field ripple is important).) The core rotation will be in the direction of the plasma current provided the $\mathrm{H}$-mode pedestal is in the high or intermediate collisionality regime. (The proportionality coefficient between $U_{\theta, \mathrm{i}}$ and $T_{\mathrm{i}}^{\prime}$ indeed changes sign in the low collisionality regime [14].) The excellent agreement between theory and experiment has therefore the following consequences:

(a) in the ELM-free Ohmic H-mode discharge discussed in [11], edge plasma ions indeed behave according to neoclassical (or, rather, sub-neoclassical) predictions; they are moreover in the high collisionality regime, for neoclassical heat losses would otherwise exceed the Ohmic power input;

(b) at the LCFS, the radial electric field is necessarily negative if the toroidal velocity vanishes (the latter is a reasonable assumption for Ohmic discharges), as shown by the radial momentum balance equation in the high collisionality regime;

(c) in H-mode discharges, anomalous Reynolds stresses and anomalous viscosity in the plasma core, if any, have a negligible impact on the toroidal rotation.

Another important outcome of the comparison between theory and experiment concerns an H-mode discharge with formation of an internal transport barrier (ITB) and concomitant impurity accumulation [15]. An extension of the theory developed in [12] (an extension which had already then been deemed necessary under the following circumstances) shows that the large neoclassical outward main ion radial flow now necessary to balance the impurity influx should lead, as observed, to a reduction of the toroidal rotation velocity in the core. Spin down and spin up on the energy confinement timescale at the $\mathrm{H}-\mathrm{L}$ and $\mathrm{L}-\mathrm{H}$ transitions as well as during ELMs can be explained similarly (although particle and energy transport is here anomalous).

Fitting the toroidal and poloidal rotation velocities by products of powers of the local plasma parameters is possible only if the width of the temperature pedestal satisfies a certain scaling relation, which is obtained. Consequences of this hypothesis are compared to some experimental results [16].

In the pedestal of the ELM-free discharge discussed in [11], the product of the density length scale with the toroidal velocity gradient predicted by theory is close to the sound speed. This implies that the Kelvin-Helmholtz $(\mathrm{K}-\mathrm{H})$ parallel velocity shear (PVS) instability [17] is close to being excited and clearly suggests that the latter is the triggering mechanism for the transition from ELM-free to enhanced $\mathrm{D}_{\alpha}$ (EDA) operation [18]. The properties of the K-H PVS instability are actually consistent with the 'quasi-coherent' mode observed in all EDA discharges and with the edge safety factor being the decisive parameter for the transition from ELM-free to EDA $\mathrm{H}$-modes at $q_{\mathrm{s}} \cong 3.5-4$.

Charge-exchange neutrals will slow down the toroidal rotation above a critical density, which is estimated.

The paper is organized as follows. The relevant experimental results are summarized in section 2 . The theoretical model is described in section 3 and the analytical and numerical procedures of integration in section 4 . The theoretical velocity and radial electric field profiles for the ELM-free Ohmic H-mode discharge are obtained in section 5.1; the suppression of the core toroidal rotation after formation of an ITB leading to impurity accumulation and its 
spin down or spin up after an $\mathrm{H}-\mathrm{L}$ or an $\mathrm{L}-\mathrm{H}$ transition or in association with an ELM are discussed in sections 5.2 and 5.3, respectively; power scaling laws are the object of section 5.4. Sub-neoclassical energy transport is estimated and compared to the input power in section 6. Our results are summarized in section 7, where we also discuss the roles of charge-exchange neutrals and divertor geometry, show that the counter rotation velocity observed in some other tokamaks is compatible with an extension of the theory to the low collisionality regime, and note that the PVS $\mathrm{K}-\mathrm{H}$ instability is near its instability threshold in the region of the pedestal. A preliminary version of this work was presented in [19].

\section{Summary of experimental results}

The experimental results presented here were obtained from the Alcator C-Mod tokamak, a compact (major radius $R=0.67 \mathrm{~m}$, typical minor radius of $0.22 \mathrm{~m}$ and elongation $\kappa<1.8$ ), high magnetic field device $\left(2.5 \mathrm{~T}<B_{\mathrm{T}}<8 \mathrm{~T}\right)$ in the lower single null configuration. The plasma current is driven in the direction of the toroidal magnetic field. Although $5 \mathrm{MW}$ of ICRF heating power are available, the scope of this paper will only include purely Ohmic plasmas. High spatial resolution electron temperature profiles have been obtained with an edge Thomson scattering system (spatial resolution of $1 \mathrm{~mm}$ ) and from electron cyclotron emission. Similarly, electron density profiles have been obtained by edge Thomson scattering and from the visible continuum using a high spatial resolution $(1.5 \mathrm{~mm})$ imaging CCD system. For high-density Alcator C-Mod discharges, the edge ion temperature profile (not measured) is taken to be the same as the electron temperature profile. Representative edge electron temperature and density profiles are shown in figure 1, for an Ohmic $\mathrm{H}$-mode discharge from [11]. Core toroidal rotation velocities have been obtained from the Doppler shifts of argon x-ray lines and, when available, from sawtooth precursors from magnetic measurements [20]. For Ohmic L-mode discharges, the central

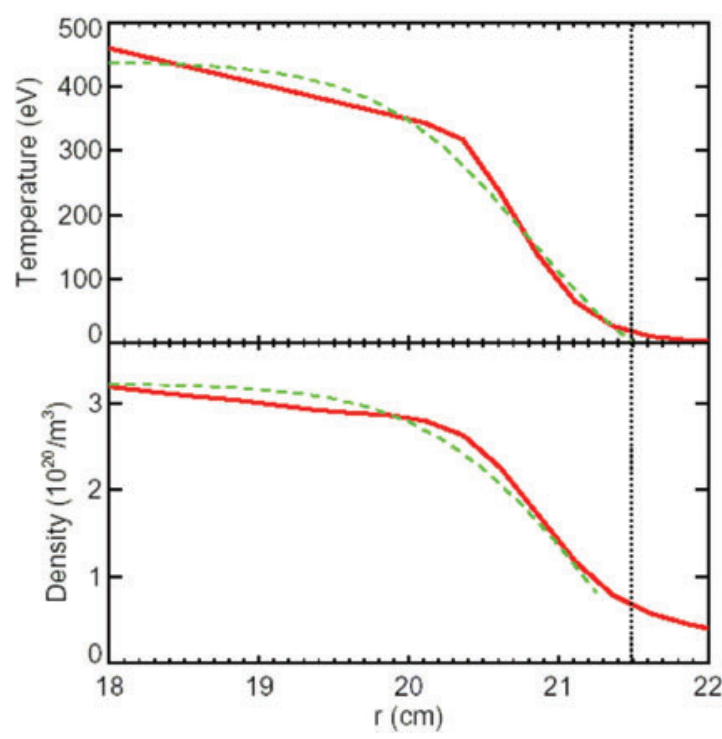

Figure 1. Comparison between the measured ( - ) and model (- - - ) temperature and density profiles in the pedestal of the reference discharge [11]. impurity rotation velocity is small, typically $<10^{4} \mathrm{~m} \mathrm{~s}^{-1}$, and in the counter current direction [21]. During Ohmic H-modes, strong edge pedestals appear (figure 1) and the core toroidal rotation velocity increases to values as large as $6 \times 10^{4} \mathrm{~m} \mathrm{~s}^{-1}$, in the co-current direction $[20,11]$, in the absence of any external momentum input. The timescale for the spin up in the plasma core after the $\mathrm{H}$-mode transition (and the spin down after the $\mathrm{H}-\mathrm{L}$ transition) is of the order of the energy confinement time, about $50 \mathrm{~ms}$. The edge pedestals appear and collapse on a much shorter timescale, of order $5 \mathrm{~ms}$. The rotation velocity during the $\mathrm{H}$-mode is proportional to the increase in the plasma stored energy following the H-mode transition, and inversely proportional to the plasma current $[20,11,15]$. Very similar behaviour is observed during ICRF-induced H-modes [15]. Some H-mode plasmas undergo ITB formation, which occurs in conjunction with a decrease and reversal of the core toroidal rotation velocity [15].

\section{Description of the theoretical model}

Since the gradient of the toroidal velocity is proportional to $U_{\theta, \mathrm{i}} \partial_{r} \ln T_{\mathrm{i}}$ and, in the $\mathrm{H}$-mode, the temperature gradient is much larger in the edge region than in the plasma core-so that the latter does not contribute significantly to the toroidal velocity (cf section 1) - it will be sufficient in the following to approximate the temperature profile by

$$
T_{\mathrm{i}}(r)=T_{\mathrm{i}}\left(r_{\mathrm{inf}}\right)\left[1-\frac{\tanh \left(r-r_{\text {inf }}\right)}{\Delta_{T}}\right]
$$

(see figure 1). In the equatorial plane, the radius of the pedestal's inflexion point of the reference discharge [11] is $r_{\text {inf }} \cong 20.8 \times 10^{-2} \mathrm{~m}$ and the local temperature gradient scale $\Delta_{T} \cong 0.6 \times 10^{-2} \mathrm{~m}$. For convenience, we further approximate the density profile by

$$
N_{\mathrm{i}}(r) \propto\left[T_{\mathrm{i}}(r)\right]^{1 / \eta_{\mathrm{i}}},
$$

where $\eta_{\mathrm{i}} \equiv \partial_{r} \ln T_{\mathrm{i}} / \partial_{r} \ln N_{\mathrm{i}} \cong 1.6$ is considered as being constant. (That simplifying assumption does not quite reflect the observation, as the density pedestal is slightly shifted inward of the temperature pedestal; the shape of the density profile does not play a crucial role in what follows however, so that a mean value of $\eta_{\mathrm{i}}$ suffices.)

The ion temperature and density at the inflexion point are about $165 \mathrm{eV}$ and $1.87 \times 10^{20} \mathrm{~m}^{-3}$, respectively. Accordingly, the ion collision frequency $v_{\mathrm{i}}$ (as defined by Braginskii [22]) and the collisionality parameter

$$
\hat{v}_{\mathrm{i}}=\frac{q R v_{\mathrm{i}}}{c_{\mathrm{i}}}
$$

are $\cong 0.44 Z_{\text {eff }, \mathrm{i}} \times 10^{5} \mathrm{~s}^{-1}$ and $\cong 0.33 q Z_{\text {eff }, \mathrm{i}}$ at that position $\left(Z_{\text {eff }, \mathrm{i}}\right.$ is the effective charge entering the ion collision frequency in the presence of impurities, $q$ the safety factor and $c_{\mathrm{i}}=\left(T_{\mathrm{i}} / m_{\mathrm{i}}\right)^{1 / 2}$ the ion thermal velocity). According to [23], the H-mode pedestal must then be considered as being highly collisional, for $\hat{v}_{\mathrm{i}} \geqslant 0.22$. (It is difficult to determine with precision where the boundaries between the various collisionality regimes actually are; to our knowledge, [23] is the only reference where this has been attempted.) 
Furthermore, the features of edge turbulence observed in the $\mathrm{H}$-mode being compatible with unstable electron drift waves but not with ion temperature gradient modes [5], we assume anomalous ion edge transport to be negligible; that hypothesis is actually the only one which is consistent with power balance as shown in section 6 . The equations providing the toroidal and poloidal velocity profiles $U_{\varphi, \mathrm{i}}(r)$ and $U_{\theta, \mathrm{i}}(r)$ are then

$$
\begin{array}{r}
\partial_{r}\left[\eta_{2, \mathrm{i}}\left(\frac{\partial U_{\varphi, \mathrm{i}}}{\partial r}-\frac{0.107 q^{2}}{1+Q^{2} / S^{2}} \frac{\partial \ln T_{\mathrm{i}}}{\partial r} \frac{B_{\varphi}}{B_{\theta}} U_{\theta, \mathrm{i}}\right)\right. \\
\left.-m_{\mathrm{i}} N_{\mathrm{i}} U_{\varphi, \mathrm{i}} U_{r, \mathrm{i}}\right]=m_{\mathrm{i}}\left(\partial_{t}-v_{\mathrm{i}, z}+v_{c x}\right) N_{\mathrm{i}} U_{\varphi, \mathrm{i}}
\end{array}
$$

and

$$
\begin{aligned}
\frac{e B_{\varphi}}{\partial_{r} T_{\mathrm{i}}} U_{\theta, \mathrm{i}}+1.83=\frac{0.45 \Lambda^{2}}{1+Q^{2} / S^{2}} & \\
\times & {\left[\frac{e B_{\theta}}{\partial_{r} T_{\mathrm{i}}} \frac{\partial U_{\varphi, \mathrm{i}}}{\partial \ln T_{\mathrm{i}}}+\frac{1}{2}\left(\frac{e B_{\theta}}{\partial_{r} T_{\mathrm{i}}} U_{\varphi, \mathrm{i}}\right)^{2}\right.} \\
- & \frac{e B_{\theta}}{\partial_{r} T_{\mathrm{i}}} U_{\varphi, \mathrm{i}}\left(\frac{e B_{\theta}}{\partial_{r} T_{\mathrm{i}}} U_{\theta, \mathrm{i}}-\left(1+2 \eta_{\mathrm{i}}^{-1}\right)\right) \\
+ & \left.1.90\left(\frac{e B_{\theta}}{\partial_{r} T_{\mathrm{i}}} U_{\theta, \mathrm{i}}-0.8\left(1+1.6 \eta_{\mathrm{i}}^{-1}\right)\right)^{2}\right] .
\end{aligned}
$$

Here, $\eta_{2, \mathrm{i}}=1.2 N_{\mathrm{i}} T_{\mathrm{i}} v_{\mathrm{i}} / \Omega_{\mathrm{i}}^{2}$ is the classical perpendicular viscosity coefficient [22], $\Omega_{\mathrm{i}}$ the ion cyclotron frequency, $v_{c x} N_{\mathrm{i}} U_{\varphi, \mathrm{i}}$ the rate at which toroidal momentum is transferred to the walls via charge exchange neutrals, $v_{i, z}$ the neutral ionization frequency,

$$
\Lambda \equiv \frac{\hat{v}_{\mathrm{i}}\left(a_{\mathrm{i}}\right)_{\mathrm{p}}}{L_{T}}
$$

the dimensionless parameter associated with FLR effects, $L_{T} \equiv\left(\partial_{r} \ln T_{\mathrm{i}}\right)^{-1}$ the temperature gradient length scale, $\left(a_{\mathrm{i}}\right)_{\mathrm{p}}=\left(B_{\varphi} / B_{\theta}\right) a_{\mathrm{i}}$ and $a_{\mathrm{i}}=c_{\mathrm{i}} / \Omega_{\mathrm{i}}$ the Larmor radii calculated with respect to the poloidal and toroidal fields, respectively, and, finally,

$$
\frac{Q}{S}=0.51 \Lambda\left[\left(\frac{e B_{\varphi}}{\partial_{r} T_{\mathrm{i}}}\right) U_{\theta, \mathrm{i}}-0.625\left(1+2 \eta_{\mathrm{i}}^{-1}\right)\right] .
$$

Equations (4) and (5) are identical to equations (1) and (4) of [12], except for the following simplifications and respective generalization:

(a) the terms describing the effects of a polarization current and of acceleration by a beam have been left out;

(b) the effect of ion inertia on toroidal momentum transport has now been taken into account: thus the last term inside the square bracket on the left-hand side of equation (4); the term $m_{\mathrm{i}} U_{\varphi, \mathrm{i}}\left(\partial_{t}-v_{\mathrm{i}, z}\right) N_{\mathrm{i}}$ on the right-hand side balances the contribution $-m_{\mathrm{i}} U_{\varphi, \mathrm{i}} \partial_{r}\left(N_{\mathrm{i}} U_{r, \mathrm{i}}\right)$ on the left.

The role of inertia was neglected in equation (1) of [12] on the grounds that (see below equation $\left(8^{\prime}\right)$ ) 'With the ordering adopted here..., the contribution arising from the density evolution and from the radial flux of angular momentum are negligible in the framework of the neoclassical theory of a one ion species plasma.' The situation is quite different in a plasma with accumulating impurities, as is the $\mathrm{H}$-mode discharge of [15] after formation of the ITB; the reason is that neoclassical particle transport is now enhanced, being governed by the ion, instead of the electron, Larmor radius and collision frequency. This term, which corresponds to a convective transport process of toroidal momentum, will also play an essential role in the interpretation of the spin up or down observed in the plasma core in conjunction with equilibrium bifurcation.

Equation (4) also reduces to the toroidal momentum diffusion equation derived by Hinton and Wong [24] and by Connor et al [25] to describe the relaxation of initial rotation profiles satisfying the conditions $U_{\varphi, \mathrm{i}} \gg\left(B_{\varphi} / B_{\theta}\right) U_{\theta, \mathrm{i}}$ and $U_{r, \mathrm{i}}=0$. It is important to note that the momentum diffusion coefficient is classical in contradiction with earlier assertions by Hogan [26], who obtained a neoclassical enhancement factor proportional to $q^{2}$, and by Stacey [27] and Stacey and Sigmar [28], who claimed that $\eta_{2, \mathrm{i}}$ should be substituted by the much larger gyro-viscous coefficient $\eta_{4, \mathrm{i}} \cong\left(\Omega_{\mathrm{i}} / \nu_{\mathrm{i}}\right) \eta_{2, \mathrm{i}}$.

Equation (5) yields the usual neoclassical result $U_{\theta, \mathrm{i}}=$ $-1.83 \partial_{r} T_{\mathrm{i}} / e B_{\varphi}$ [14] in the limit $\Lambda \rightarrow 0$ considered in all earlier theoretical works on neoclassical transport [29, 23]. Introducing the experimental data quoted above and noting that $q=3.4, B_{\varphi}=5.2 \mathrm{~T}, \kappa=1.62$ - $\mathrm{so}$ that $B_{\theta}=$ $B_{\varphi} \kappa^{0.5} a / q R=0.625 \mathrm{~T}(a=0.215 \mathrm{~m}$ is the plasma minor radius in the equatorial plane; the effective minor radius is $\left.\kappa^{0.5} a\right)$ - and $L_{T}\left(r_{\text {inf }}\right)=\kappa^{0.5} \Delta_{T}$, we estimate $\Lambda \cong 0.43 Z_{\text {eff }, \mathrm{i}}$ at the inflexion point of the temperature pedestal of the discharge discussed in [11]. Under these conditions, the poloidal velocity at the edge is considerably different from that predicted by the conventional theory due to FLR effects: in this respect it is worth noting that the ratio $\left(a_{\mathrm{i}}\right)_{\mathrm{p}} / L_{T} \cong 0.38$ at $r=r_{\text {inf }}$ $\left(\left(a_{\mathrm{i}}\right)_{\mathrm{p}} \cong 0.29 \mathrm{~cm}\right)$.

Equation (4) can readily be integrated from the magnetic axis (where $U_{r, \mathrm{i}} \equiv \partial_{r} \equiv 0$; we assume that the diffusive character of the equation remains valid in the core, where viscosity might, however, be anomalous) up to the edge coordinate $r$; thus

$$
\begin{aligned}
& \partial_{r}\left(u_{\varphi, \mathrm{i}} \partial_{r} T_{\mathrm{i}}\right)-m_{\mathrm{i}} \eta_{2, \mathrm{i}}^{-1} N_{\mathrm{i}} U_{r, \mathrm{i}} u_{\varphi, \mathrm{i}} \partial_{r} T_{\mathrm{i}} \\
&= 0.107 q^{2}\left(1+\frac{Q^{2}}{S^{2}}\right)^{-1}\left(u_{\theta, \mathrm{i}} \partial_{r} T_{\mathrm{i}}\right) \partial_{r} \ln T_{\mathrm{i}} \\
&+e B_{\theta} \eta_{2, \mathrm{i}}^{-1} m_{\mathrm{i}} \int_{0}^{r}\left(\partial_{t}-v_{\mathrm{i}, z}+v_{c x}\right) N_{\mathrm{i}} U_{\varphi, \mathrm{i}} \mathrm{d} r^{\prime},
\end{aligned}
$$

where $u_{\varphi, \mathrm{i}}=\left(e B_{\theta} / \partial_{r} T_{\mathrm{i}}\right) U_{\varphi, \mathrm{i}}$ and $u_{\theta, \mathrm{i}}=\left(e B_{\varphi} / \partial_{r} T_{\mathrm{i}}\right) U_{\theta, \mathrm{i}}$ are suitably normalized toroidal and poloidal velocities.

Equation (8) can further be integrated to yield

$$
\begin{aligned}
& \left(u_{\varphi, \mathrm{i}} \partial_{r} T_{\mathrm{i}}\right)_{r_{\mathrm{s}}}^{r}=\int_{r_{\mathrm{s}}}^{r} \exp \left(\int_{r^{\prime}}^{r} \eta_{2, \mathrm{i}}^{-1} m_{\mathrm{i}} N_{\mathrm{i}} U_{r, \mathrm{i}} \mathrm{d} r^{\prime \prime}\right) \\
& \times\left[0.107 q^{2}\left(1+\frac{Q^{2}}{S^{2}}\right)^{-1}\left(u_{\theta, \mathrm{i}} \partial_{r^{\prime}} T_{\mathrm{i}}\right) \partial_{r^{\prime}} \ln T_{\mathrm{i}}\right. \\
& \left.+e B_{\theta} \eta_{2, \mathrm{i}}^{-1} m_{\mathrm{i}} \int_{0}^{r^{\prime}}\left(\partial_{t}-v_{\mathrm{i}, z}+v_{c x}\right) N_{\mathrm{i}} U_{\varphi, \mathrm{i}} \mathrm{d} r^{\prime \prime}\right] \mathrm{d} r^{\prime},
\end{aligned}
$$

where $r_{\mathrm{s}}$ is the radius of the LCFS. It is appropriate recalling at this point that equations (4) and (5) of [12] were derived taking into account that the variation length scales $\left(L_{\perp}\right)$ of density, temperature, rotation velocities and radial electric field are, at the plasma edge of $\mathrm{H}$-mode discharges, much smaller than those $(\sim r$ or $R$ ) of the magnetic field components and of the safety factor. 


\section{Integration of the system of equations}

The detailed evaluations related to the stationary reference discharge discussed in [11] are made assuming negligible radial particle flow velocity $\left(U_{r, \mathrm{i}}=0\right)$ as well as negligible ionization and momentum loss rates. Inserting $\partial_{r} U_{\varphi, \mathrm{i}}$ (equation (8)) into (5) yields, under these conditions,

$$
\begin{aligned}
u_{\theta, \mathrm{i}}[1 & \left.+0.048 q^{2} \Lambda^{2}\left(1+\frac{Q^{2}}{S^{2}}\right)^{-2}\right]+1.83 \\
= & 0.45 \Lambda^{2}\left(1+\frac{Q^{2}}{S^{2}}\right)^{-1}\left\{0.5 u_{\varphi, \mathrm{i}}^{2}-u_{\varphi, \mathrm{i}}\left[u_{\theta, \mathrm{i}}-\left(1+2 \eta_{\mathrm{i}}^{-1}\right)\right]\right. \\
& \left.+1.90\left[u_{\theta, \mathrm{i}}-0.8\left(1+1.6 \eta_{\mathrm{i}}^{-1}\right)\right]^{2}\right\} .
\end{aligned}
$$

\subsection{Boundary conditions}

Equation $\left(8^{\prime}\right)$ provides the toroidal velocity in the plasma relative to its value at the LCFS; information on the latter is thus required. In the absence of precise experimental information, we shall assume $U_{\varphi, \mathrm{i}}\left(r_{\mathrm{s}}\right)=0$ for Ohmic discharges.

The edge gradients being steep and the position of the LCFS unknown within $\cong 2-3 \mathrm{~mm}$, there is a considerable uncertainty on the LCFS values of the temperature $T_{\mathrm{i}, \mathrm{s}} \equiv T_{\mathrm{i}}\left(r_{\mathrm{s}}\right)$ and density $N_{\mathrm{i}, \mathrm{s}} \equiv N_{\mathrm{i}}\left(r_{\mathrm{s}}\right)$. This affects, in turn, the value $\Lambda_{\mathrm{s}}$ of the parameter $\Lambda$ at $r_{\mathrm{s}}$ which further suffers from uncertainties on the profile of $Z_{\text {eff }, i}$; obviously, the latter cannot indeed be resolved on the scale of the pedestal width. Since the poloidal velocity depends on $\Lambda$ (cf equation (9)), we investigate, under these circumstances, whether one of the following hypotheses is compatible with experimental data:

(a) the radial electric field vanishes at the LCFS;

(b) the poloidal velocity $U_{\theta, \mathrm{i}}$ vanishes at the LCFS

Since $\left(u_{\varphi, \mathrm{i}}\right)_{\mathrm{s}}=0$ by hypothesis, equation (9) yields

$$
\begin{aligned}
\left(u_{\theta, \mathrm{i}}\right)_{\mathrm{s}} & +1.83=\frac{\Lambda_{\mathrm{s}}^{2}}{1+\left(Q^{2} / S^{2}\right)_{\mathrm{s}}} \\
\times & {\left[0.855\left[\left(u_{\theta, \mathrm{i}}\right)_{\mathrm{s}}-0.8\left(1+1.6 \eta_{\mathrm{i}}^{-1}\right)\right]^{2}\right.} \\
- & \left.\frac{0.048 q^{2}\left(u_{\theta, \mathrm{i}}\right)_{\mathrm{s}}}{1+\left(Q^{2} / S^{2}\right)_{\mathrm{s}}}\right]
\end{aligned}
$$

at $r_{\mathrm{s}}$. Noting that

$$
\frac{Q}{S}=0.51 \Lambda\left[u_{\theta, \mathrm{i}}-0.625\left(1+2 \eta_{\mathrm{i}}^{-1}\right)\right]
$$

for all $r$ values, we are led to the following conclusions.

(a) The hypothesis $E_{r}\left(r_{\mathrm{s}}\right)=0$ would imply $\left(u_{\theta, \mathrm{i}}\right)_{\mathrm{s}}=$ $\left(1+\eta_{\mathrm{i}}^{-1}\right)$, in view of the radial momentum balance equation. Accordingly, $\Lambda_{\mathrm{s}}^{2}$ would be one of the solutions of

$$
\begin{aligned}
\Lambda_{\mathrm{s}}^{2}= & \left(2.83+\eta_{\mathrm{i}}^{-1}\right)\left\{1+\left[0.51\left(0.375-0.25 \eta_{\mathrm{i}}^{-1}\right)\right]^{2} \Lambda_{\mathrm{s}}^{2}\right\} \\
& \times\left[0.855\left(0.2-0.28 \eta_{\mathrm{i}}^{-1}\right)^{2}\right. \\
& \left.-\frac{0.048 q^{2}\left(1+\eta_{\mathrm{i}}^{-1}\right)}{1+\left[0.51\left(0.375-0.25 \eta_{\mathrm{i}}^{-1}\right)\right]^{2} \Lambda_{\mathrm{s}}^{2}}\right]^{-1}
\end{aligned}
$$

The right-hand side being negative for $\Lambda_{\mathrm{s}}^{2}<2 \times 10^{5}$ if $\eta_{\mathrm{i}}=1.6$, we conclude that the hypothesis that the radial electric field vanishes at the LCFS must be rejected. We note also that equation (10) cannot be satisfied in the limit $\Lambda_{\mathrm{s}} \rightarrow 0$ if $E_{r}\left(r_{\mathrm{s}}\right)=0$; the 'conventional' neoclassical theory indeed yields $u_{\theta, \mathrm{i}}=-1.83$, which is incompatible with $u_{\theta, \mathrm{i}}=\left(1+\eta_{\mathrm{i}}^{-1}\right)$.

(b) If the poloidal velocity $U_{\theta, \mathrm{i}}$ vanishes at the LCFS, the value of $\Lambda_{\mathrm{s}}^{2}$ must be, according to equation (10) where $\eta_{\mathrm{i}}=1.6$;

$$
\begin{aligned}
\Lambda_{\mathrm{s}}^{2} & =\left[0.299\left(1+1.6 \eta_{\mathrm{i}}^{-1}\right)^{2}-0.102\left(1+2 \eta_{\mathrm{i}}^{-1}\right)^{2}\right]^{-1} \\
& \cong 1.47
\end{aligned}
$$

This turns out to be compatible, within error bars, with the measured temperature and density at the LCFS if $Z_{\mathrm{eff}, \mathrm{i}}\left(r_{\mathrm{s}}\right)=1$. We shall assume $U_{\theta, \mathrm{i}}\left(r_{\mathrm{s}}\right)=0$ and $\Lambda_{\mathrm{s}}^{2}=1.47$ in the following: we note that this implies, on the grounds of a continuity argument, that the parallel flow is directed symmetrically into both divertor legs beyond the LCFS.

\subsection{Analytical evaluation}

We shall approximate the solution of equation (9) in the range $\left(\Lambda^{2}=0\right.$ (corresponding to the core); $\Lambda^{2}=\Lambda_{\mathrm{s}}^{2}=$ 1.47 (corresponding to the LCFS)) by the linear interpolation formula

$$
u_{\theta, \mathrm{i}}=-1.83\left(1-\frac{\Lambda^{2}}{\Lambda_{\mathrm{s}}^{2}}\right) .
$$

The denominator $1+Q^{2} / S^{2}$ is now a cubic function of $\Lambda^{2}$ which will be replaced by a quadratic form for the purpose of the analytical integration in $\left(8^{\prime}\right)$; thus, defining $f=\Lambda / \Lambda_{\mathrm{s}}$, one has

$$
1+\frac{Q^{2}}{S^{2}}=1+\alpha f^{2}+\beta f^{4}
$$

where $\alpha$ and $\beta$ are obtained by matching (14) to the exact expression obtained from (7) for $\Lambda=\Lambda_{\mathrm{s}}$ and $\Lambda=\Lambda_{\mathrm{M}}$, the latter corresponding to the maximum value. Thus

$$
\alpha=0.87 \Lambda_{\mathrm{s}}^{2} f_{\mathrm{M}}^{2}\left(9 f_{\mathrm{M}}^{2}-1\right)
$$

and

$$
\beta=-0.87 \Lambda_{\mathrm{s}}^{2}\left(5 f_{\mathrm{M}}^{2}-1\right),
$$

where

$$
f_{\mathrm{M}}^{2}=\frac{1}{3}\left[1+\left(\frac{0.625}{1.83}\right)\left(1+2 \eta_{\mathrm{i}}^{-1}\right)\right] .
$$

We must finally specify the analytical form of the function $\Lambda^{2}(r)$. Assuming $Z_{\mathrm{eff}, \mathrm{i}}(r) \propto\left[T_{\mathrm{i}}(r)\right]^{z}$, we have

$$
\begin{aligned}
\Lambda^{2}(r) & =\Lambda_{\text {inf }}^{2}\left(\frac{T_{\mathrm{i}}}{T_{\mathrm{i}, \text { inf }}}\right)^{-2(c+1)}\left[\frac{\partial_{r} T_{\mathrm{i}}}{\left(\partial_{r} T_{\mathrm{i}}\right)_{\mathrm{inf}}}\right]^{2} \\
& =\Lambda_{\mathrm{inf}}^{2} \hat{T}^{-2 c}(2-\hat{T})^{2},
\end{aligned}
$$

where $T_{\mathrm{i}, \text { inf }}$ is the value of $T_{\mathrm{i}}$ at the pedestal inflexion point, $\hat{T}=T_{\mathrm{i}} / T_{\mathrm{i}, \text { inf }}$ and

$$
2 c=3-2 \eta_{\mathrm{i}}^{-1}-2 z
$$

(we note that $\left.L_{T}=-\kappa^{0.5} \Delta(2-\hat{T})^{-1}\right)$. The integral in $\left(8^{\prime}\right)$ can be performed easily if $c=0$; indeed, $\left(\partial_{r} \ln T_{\mathrm{i}}\right) \mathrm{d} T \propto \mathrm{d} f^{2}$ 
in that case, so that

$$
\begin{aligned}
u_{\varphi, \mathrm{i}} \partial_{r} & T_{\mathrm{i}}=\frac{0.196 q^{2}}{2\left(\alpha^{2}-4 \beta\right)^{1 / 2}} \frac{\Lambda_{\mathrm{s}}^{2}}{\Lambda_{\mathrm{inf}}^{2}}\left(\partial_{r} T_{\mathrm{i}}\right)_{\mathrm{inf}} \\
\times & {\left[\ln \left[\frac{\Lambda_{\mathrm{inf}}^{2}}{\Lambda_{\mathrm{s}}^{2}}(2-\hat{T})^{2}-a_{-}\right]^{1-a_{-}}\right.} \\
\times & {\left[a_{+}-\frac{\Lambda_{\mathrm{inf}}^{2}}{\Lambda_{\mathrm{s}}^{2}}(2-\hat{T})^{2}\right]^{a_{+}-1} } \\
- & \left.\ln \left(1-a_{-}\right)^{1-a_{-}}\left(a_{+}-1\right)^{a_{+}-1}\right],
\end{aligned}
$$

where

$$
a_{\mp}=\frac{-\alpha \pm\left(\alpha^{2}-4 \beta\right)^{1 / 2}}{2 \beta} \text {. }
$$

\subsection{Numerical evaluation}

The full time-dependent equations (4) and (5) have also been solved numerically, without introducing any simplification, but retaining the form $(16 a)$ of $\Lambda^{2}(r)$. Comparing with the above analytical result for $c=0$ has allowed validation of both the numerical and the approximate analytical schemes.

\section{Determination of the velocity and electric field profiles}

\subsection{H-mode discharge without ITB}

Introducing $\eta_{\mathrm{i}}=1.6$ and $\Lambda_{\mathrm{s}}^{2}=1.47$ in equations $(15 a)-(15 d)$, we find successively $f_{\mathrm{M}}^{2}=0.59, \alpha=3.25, \beta=-2.49$, $a_{-}=-0.26$ and $a_{+}=1.56$. Due to the large ion to electron mass ratio, the effective charge entering the ion collision frequency in the presence of impurities,

$$
Z_{\text {eff }, \mathrm{i}}=1+\sqrt{2} \sum_{I}\left(\frac{N_{I}}{N_{\mathrm{i}}}\right) Z_{I}^{2},
$$

is larger than the effective charge appropriate to the electrical resistivity,

$$
Z_{\text {eff,e }}=1+\sum_{I}\left(\frac{N_{I}}{N_{\mathrm{e}}}\right) Z_{I}^{2} .
$$

The latter is found experimentally to be $\cong 1.4$; we assume, therefore, $Z_{\text {eff }, \mathrm{i}}\left(r_{\text {inf }}\right)=1.57$ and $\Lambda_{\text {inf }}^{2}=\left(0.43 Z_{\text {eff }, \mathrm{i}}\right)^{2}=0.46$. Equation $(16 a)$ then yields $\hat{T}_{\mathrm{i}, \mathrm{s}}=2-\Lambda_{\mathrm{s}} / \Lambda_{\text {inf }}=0.21$ if $c=0$; thus $T_{\mathrm{i}, \mathrm{s}}=35 \mathrm{eV}$, which corresponds roughly to the value measured at the radius of the nominal LCFS. Introducing $q=3.4$ and the ratio $\Lambda_{\text {inf }}^{2} / \Lambda_{\mathrm{s}}^{2}=0.46 / 1.47=0.31$ in equation (17) yields the toroidal velocity profile marked by squares in figure $2(b)$. (The temperature profile, equation (1), is given in figure $2(a)$ up to the radius where $U_{\theta, \mathrm{i}}=0$ assumed to correspond to the LCFS); in particular, the velocity in the core (where $\hat{T}=2$ ) predicted by the approximate analytical model is

$$
U_{\varphi, r}(r \rightarrow 0)=1.14 \frac{\left(T_{\mathrm{i}, \mathrm{inf}} / e B_{\theta}\right)}{\Lambda \sqrt{\kappa}}=38 \mathrm{~km} \mathrm{~s}^{-1} .
$$

Numerical integration of the exact equations (9) and ( $\left(8^{\prime}\right)$ yields, still for $c=0$, the profile marked by triangles; here,

$$
U_{\varphi, \mathrm{i}}(r \rightarrow 0)=34 \mathrm{~km} \mathrm{~s}^{-1} \text {. }
$$
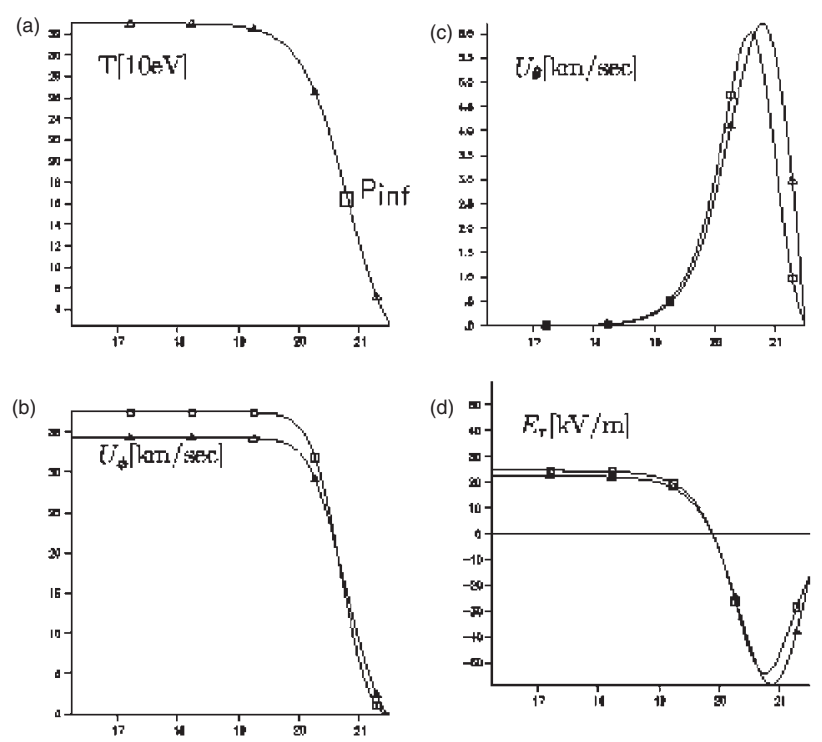

Figure 2. Edge profiles of the toroidal and poloidal velocity components and of the radial electric field predicted by theory, assuming that the dimensionless parameter $\Lambda^{2}$ is of the form (16a) with $c=0$; the model ion temperature profile (equation (1)) is also shown; the position of the LCFS corresponds to $U_{\theta, \mathrm{i}}=0$. Triangles correspond to the exact numerical solution of the full equations (9) and $\left(8^{\prime}\right)$, squares to the simplified analytical estimates (17) and (13).

Both results agree quite well with the measured value of about $35 \mathrm{~km} \mathrm{~s}^{-1}$ and are, as observed, in the co-current direction. Figure 2(c) shows the poloidal velocities according to the interpolation formula (13) and the exact numerical integration. Finally, figure $2(d)$ shows the corresponding radial electric fields

$$
E_{r}=B_{\theta} U_{\varphi, \mathrm{i}}-B_{\varphi} U_{\theta, \mathrm{i}}+\left(e N_{\mathrm{i}}\right)^{-1} \partial_{r} P_{\mathrm{i}} .
$$

The excellent agreement between the two sets of curves confirms the validity of both methods.

According to equation $(16 b), c=0$ and $\eta_{\mathrm{i}}=1.6$ correspond to $z=0.875$; this leads to a value of $Z_{\mathrm{eff}, \mathrm{i}}$ smaller than unity at the LCFS if, indeed, $Z_{\text {eff, }}\left(r_{\mathrm{s}}\right)=1.57$. The numerical solution of the equations has thus been extended to the case $c=0.24$ to which correspond $z=0.635$ and $Z_{\mathrm{eff}, \mathrm{i}}\left(r_{\mathrm{s}}\right)=1$; the results are shown in figure 3 . Inside of the temperature pedestal, the toroidal velocity now reaches asymptotically the value

$$
U_{\varphi, \mathrm{i}}(r \rightarrow 0)=29 \mathrm{~km} \mathrm{~s}^{-1},
$$

still in good agreement with experiment. The temperature $T_{\mathrm{i}, \mathrm{s}}$ is, however, $81 \mathrm{eV}$ in that case, i.e. about a factor 2 larger than that reported $(\cong 40 \mathrm{eV})$ at the nominal LCFS. As mentioned earlier, the position of the latter cannot be defined with an accuracy better than $2-3 \mathrm{~mm}$. Within the framework of the hyperbolic tangent model (equation (1)), $T_{\mathrm{i}, \mathrm{s}}=40 \mathrm{eV}$ and $T_{\mathrm{i}, \text { inf }}=165 \mathrm{eV}$ correspond to a distance $r_{\mathrm{s}}-r_{\mathrm{inf}}=5.9 \mathrm{~mm}$ in the equatorial plane if $\Delta=6 \mathrm{~mm}$ whereas $T_{\mathrm{i}, \mathrm{s}}=80 \mathrm{eV}$ correspond to $r_{\mathrm{s}}-r_{\text {inf }}=3.4 \mathrm{~mm}$. The difference between these two values is within the error bars and does not allow invalidation of the prescription (12) for $\Lambda_{\mathrm{s}}^{2}$. (We recall that the latter is a consequence of the boundary condition $U_{\theta, \mathrm{i}}\left(r_{\mathrm{s}}\right)=0$ that we assumed on the ground that the flow beyond the LCFS should be directed into both divertor legs.) 

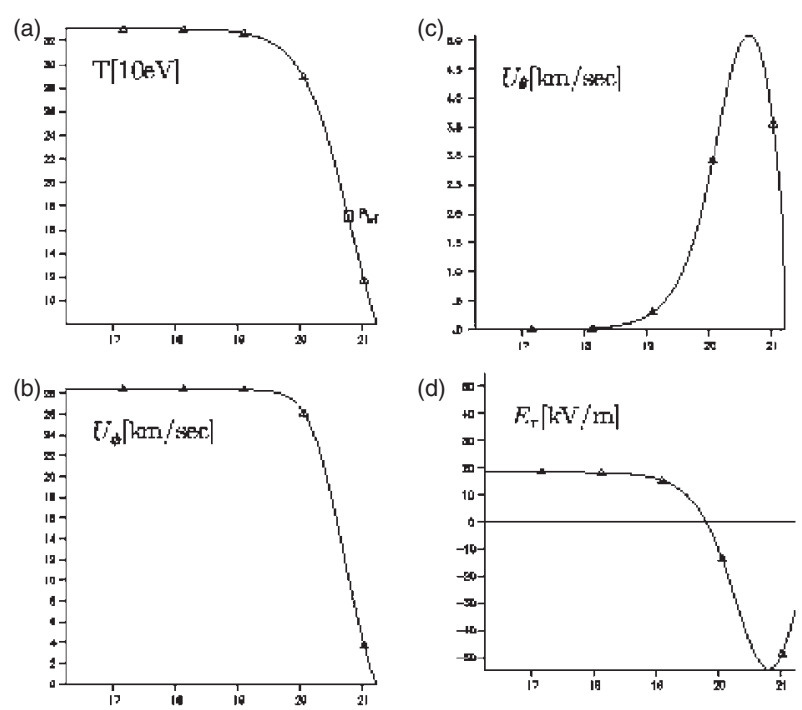

Figure 3. Exact numerical solution of equations (4) and (5) with $\Lambda^{2}$ given by (16a) with $c=0.24$; this value of $c$ corresponds $Z_{\mathrm{eff}, \mathrm{i}}=1$ at the LCFS.

The numerical results could also be optimized by varying the fitting parameters $\eta_{\mathrm{i}}$ and $\Delta$ within an acceptable range and a larger value of $Z_{\mathrm{eff}, \mathrm{i}}\left(r_{\mathrm{inf}}\right)$ would lead to a smaller value of $T_{\mathrm{i}, \mathrm{s}}$ (keeping $Z_{\mathrm{eff}, \mathrm{i}}\left(r_{\mathrm{s}}\right)$ unchanged). In any case, the assumption on the value of the poloidal velocity at the boundary is by no means essential for demonstrating that the theory of [12] is able to explain the toroidal velocity measured in Alcator C-Mod [11]; it is rather to be seen as a test that the value of the poloidal velocity at the LCFS may be imposed by physical processes.

The radial electric field just inside the separatrix has been inferred from measurements of plumes of carbon radiation emanating from a gas injection tube mounted on a reciprocating probe [30]. For an Ohmic discharge with parameters similar to that shown in figure $1, E_{r}$ at $5 \mathrm{~mm}$ inside of the nominal separatrix was found to change from $+4 \mathrm{kV} \mathrm{m}^{-1}$ during L-mode to $-17 \mathrm{kV} \mathrm{m}^{-1}$ at the beginning of the $\mathrm{H}$-mode period. These observations are in agreement with the calculations shown in figures $2(d)$ and $3(d)$. Most interestingly, the electric field profiles shown in figures $2(d)$ and $3(d)$ resemble closely these measured in DIII-D H-mode discharges [13,3] with neutral beam injection.

\subsection{H-mode discharge with ITB}

To describe adequately the toroidal rotation in a plasma with accumulating impurities, the radial flux of angular momentum which appears in equation $\left(8^{\prime}\right)$ of [12] must be retained due to the enhanced main ion outward flow; this gives rise to the exponential weight factor appearing in the integrand of equation $\left(8^{\prime}\right)$ of this paper. The variable $r^{\prime}$ being in the interval $r<r^{\prime}<r_{\mathrm{s}}$, ion inertia thus leads to a reduction of the toroidal velocity if $U_{r, \mathrm{i}}>0$, as must be the case in the H-mode discharge of [15] after formation of the ITB since the observed core electron density increase does not compensate for the positive charge increase associated with impurity accumulation. The reduction of the core toroidal rotation velocity will be important if

$$
-\int_{r_{\mathrm{inf}}}^{r \ll r_{\mathrm{inf}}} \eta_{2, \mathrm{i}}^{-1} m_{\mathrm{i}} N_{\mathrm{i}} U_{r, \mathrm{i}} \mathrm{d} r^{\prime} \approx \frac{U_{r, \mathrm{i}} \kappa^{0.5} \Delta}{1.2 a_{\mathrm{i}}^{2} v_{\mathrm{i}}} \geqslant 1,
$$

where the various factors can, for example, be evaluated at the inflexion point of the temperature pedestal. Introducing $v_{\mathrm{i}}=0.69 \times 10^{5} \mathrm{~s}^{-1}$ and $a_{\mathrm{i}}=0.35 \times 10^{-3} \mathrm{~m}$, this condition then reduces to $U_{r, \mathrm{i}}>1.33 \mathrm{~m} \mathrm{~s}^{-1}$ (to which corresponds a timescale $\kappa^{0.5} a / U_{r, \mathrm{i}}$ shorter than $13 \mathrm{~ms}$ ). As a point of comparison, the experimental value of the edge radial velocity estimated from the rate of electron accumulation in the core is $U_{r, \mathrm{e}} \cong-0.2 \mathrm{~m} \mathrm{~s}^{-1}$.

These considerations show that our theoretical model can explain the collapse of the central toroidal rotation velocity measured in Ohmic H-mode discharges after formation of an ITB, if the related impurity accumulation rate leads to a sufficiently large outward deuterium flux. For a more precise analysis, one must be aware that the contribution arising from the evolution of the density can be neglected in equation $\left(8^{\prime}\right)$ only to the extent that the gradient of the radial particle flux is also negligible; only the pedestal region is concerned here, since the drive of the toroidal rotation originates there.

\subsection{Toroidal spin down and spin up associated with $H-L$ and $L-H$ transitions and with ELMs}

In Alcator C-Mod, the timescales of toroidal rotation spin up and spin down after the $\mathrm{L}-\mathrm{H}$ and $\mathrm{H}-\mathrm{L}$ transitions are of the order of $50 \mathrm{~ms}$, which is comparable to the energy confinement time. As in section 5.2, these observations can be explained on the basis of equation (4), taking the role of ion inertia into account. Different scenarios can be envisaged for the H-mode collapse or its development. One might assume, for example, that (i) the $\mathrm{H}-\mathrm{L}$ transition is triggered by impurity accumulation and enhanced radiation in the core leading to progressive erosion, from core to edge, of the energy and density profiles, and (ii) the $\mathrm{L}-\mathrm{H}$ transition results from progressive extension of the pedestal shoulder from edge to core, after formation of the edge transport barrier. Under these circumstances, for $t>0$, equation (4) could be replaced by the following model equations:

$$
\begin{aligned}
& \left(\partial_{t}+U_{r, \mathrm{i}} \partial_{r}\right) U_{\varphi, \mathrm{i}}-D r^{-1} \partial_{r}\left(r \partial_{r} U_{\varphi, \mathrm{i}}\right) \\
& \quad=-s_{0} \partial_{r}\left[\delta\left(r-U_{r, \mathrm{i}} t\right)-\delta\left(r-r_{\mathrm{s}}\right)\right],
\end{aligned}
$$

where $U_{r, \mathrm{i}}>0$, or

$$
\begin{aligned}
& \left(\partial_{t}+U_{r, \mathrm{i}} \partial_{r}\right) U_{\varphi, \mathrm{i}}-D r^{-1} \partial_{r}\left(r \partial_{r} U_{\varphi, \mathrm{i}}\right) \\
& \quad=-s_{0} \partial_{r}\left[\delta\left(r-r_{\mathrm{s}}-U_{r, \mathrm{i}} t\right)-\delta\left(r-r_{\mathrm{s}}\right)\right],
\end{aligned}
$$

where $U_{r, \mathrm{i}}<0$. The Dirac functions model the strong localization of the product $U_{\theta, \mathrm{i}} \partial_{r} \ln T_{\mathrm{i}}$,

$$
s_{0}=\int_{r=0}^{r=r_{\mathrm{s}}} 0.107 q^{2} D\left(1+\frac{Q^{2}}{S^{2}}\right)^{-1}\left(\frac{B_{\varphi} U_{\theta, \mathrm{i}}}{B_{\theta}}\right) \partial_{r} \ln T_{\mathrm{i}} \mathrm{d} r^{\prime} .
$$

we have assumed that the velocity of the energy and particle fronts are identical to the radial flow velocity, and $D=1.2 v_{\mathrm{i}} a_{\mathrm{i}}^{2}$. In equation (21), the distance between the negative erosion front and the positive edge pedestal reduces in the interval $0 \leqslant t \leqslant r_{\mathrm{s}} / U_{r, \mathrm{i}}$ until annihilation occurs; in equation (22), 
the distance between the propagating front moving from edge to centre increases from 0 to $r_{\mathrm{s}}$ in the time interval $0 \leqslant t \leqslant$ $r_{\mathrm{s}} /-U_{r, \mathrm{i}}$. For $t \leqslant 0$, the equations are, instead,

$$
\partial_{t} U_{\varphi, \mathrm{i}}-D r^{-1} \partial_{r}\left(r \partial_{r} U_{\varphi, \mathrm{i}}\right)=s_{0} \partial_{r} \delta\left(r-r_{\mathrm{s}}\right)
$$

or

$$
\partial_{t} U_{\varphi, \mathrm{i}}-D r^{-1} \partial_{r}\left(r \partial_{r} U_{\varphi, \mathrm{i}}\right)=0 .
$$

Those admit the stationary solutions $U_{\varphi, \mathrm{i}}(r)=\left(s_{0} / D\right)$ $\left[1-\Gamma\left(r-r_{\mathrm{s}}\right)\right]$ and $U_{\varphi, \mathrm{i}}(r)=0$ corresponding to the $\mathrm{H}-$ and L-modes, respectively; $\Gamma\left(r-r_{\mathrm{s}}\right)$ is the Heaviside step function.

Solving equations (21) and (22) will be left for later work, but we point out that two timescales are involved in the equations

(a) the solution of the 'homogeneous equation' (drop the right-hand side of either equation (21) or (22)) evolves on the (long) diffusion timescale $r^{2} / D t$; the latter enters the description of the relaxation of any arbitrary initial profile;

(b) the particular solution of the 'inhomogeneous equation' evolves on the timescale of the 'source term' (the righthand side of either equation (21) or (22)).

As a consequence, the timescale for core rotation spin up or spin down is closely related to the time $r_{\mathrm{s}} /\left|U_{r, \mathrm{i}}\right|$ required by the moving energy and particle fronts (those are described by the time-dependent Dirac functions) to cross the discharge; the latter is itself of the order of the energy confinement time.

One may argue, on the same grounds, that ELMs also trigger a partial or full collapse of the toroidal rotation. During all these events (L-H, H-L and ELM transitions), particle and energy transport is, however, anomalous, rather than neoclassical; this should neither modify the structure of the convection transport term $U_{r, \mathrm{i}} \partial_{r} U_{\varphi, \mathrm{i}}$ arising from ion inertia in equation (4), nor our conclusions.

\subsection{Scaling properties}

Equations $\left(8^{\prime}\right)$ and $\left(9^{\prime}\right)$ show that $u_{\theta, \mathrm{i}}$ and $u_{\varphi, \mathrm{i}}$ are complex functions of $Q^{2} / S^{2}$, and therefore of $\Lambda^{2}$ (cf equation (7)). Power scaling laws of the toroidal and poloidal rotation velocities with respect to the local plasma parameters $\left(T_{\mathrm{i}}\right)_{\mathrm{inf}}$, $\left(N_{\mathrm{i}}\right)_{\text {inf }}, q$ and $B$ can therefore be established only if $\Lambda^{2}$ is independent of the latter, which requires that

$$
\left(L_{T}\right)_{\mathrm{inf}} \propto\left[q^{2}\left(\frac{v_{\mathrm{i}}}{\Omega_{\mathrm{i}}}\right)\right]_{\mathrm{inf}} \frac{R^{2}}{a}
$$

(cf equations (6) and (3)). Equations $\left(9^{\prime}\right),\left(8^{\prime}\right)$ and the definitions $u_{\theta, \mathrm{i}}=\left(e B_{\varphi} / \partial_{r} T_{\mathrm{i}}\right) U_{\theta, \mathrm{i}}$ and $u_{\varphi, \mathrm{i}}=\left(e B_{\theta} / \partial_{r} T_{\mathrm{i}}\right) U_{\varphi, \mathrm{i}}$ then lead to

$$
U_{\theta, \mathrm{i}}(r) \propto \frac{\left(\partial_{r} T_{\mathrm{i}}\right)_{\mathrm{inf}}}{B_{\varphi}},
$$

or, equivalently,

$$
U_{\theta, \mathrm{i}}(r) \propto\left[\frac{\left(B_{\theta} / B_{\varphi}\right) c_{\mathrm{i}}}{\hat{v}_{\mathrm{i}}}\right]_{\mathrm{inf}}
$$

and

$$
U_{\varphi, \mathrm{i}}(r) \propto\left(\frac{q^{2} \partial_{r} T_{\mathrm{i}}}{B_{\theta}}\right)_{\mathrm{inf}},
$$

or, equivalently,

$$
U_{\varphi, \mathrm{i}}(r) \propto\left(\frac{q^{2} c_{\mathrm{i}}}{\hat{v}_{\mathrm{i}}}\right)_{\mathrm{inf}} .
$$

In equations (25)- $\left(26^{\prime}\right)$, the proportionality coefficients are functional of the pedestal $\hat{T}(r)$ and $\hat{N}(r)$ only. Equation (24) implies that $\Delta_{T}$ is not an independent parameter in equation (1), but rather is related to $T_{\text {inf }}, N_{\text {inf }}$, etc. We stress that the above considerations and conclusions assume that the poloidal and toroidal velocities verify power scaling laws.

In ICRF-heated discharges, it has been observed that the pedestal temperature $\left(T_{\text {ped }} \cong 2 T_{\text {inf }}\right)$ and density are proportional to $P^{0.4}$ and $\left(N_{\mathrm{e}, L} / B\right)^{0.4} I$, respectively, $P$ being the power flowing through the scrape layer [16]. Ohmic discharges have similar properties; here, moreover, $P \propto I$ if the loop voltage is invariant. Under those conditions, equations (24) and (26) lead to

$$
\left[L_{T}\right]_{\mathrm{inf}} \propto(q R)^{1.6} a^{-0.2}\left(N_{\mathrm{e}, \mathrm{L}}\right)^{0.4} Z_{\mathrm{eff}, \mathrm{i}} A_{\mathrm{i}} B^{-1}
$$

and

$$
R U_{\varphi, \mathrm{i}} \propto\left(\frac{q}{A_{\mathrm{i}} Z_{\mathrm{eff}, \mathrm{i}}}\right)\left(\frac{B}{N_{\mathrm{e}, L}}\right)^{0.4},
$$

where $A_{\mathrm{i}}$ is the main ion atomic mass. The latter result, equation $\left(26^{\prime \prime}\right)$, agrees well with other observations from Alcator C-Mod showing that the toroidal rotation velocity measured in the $\mathrm{H}$-mode is approximately inversely proportional to the plasma current (figure 5 of [9]). The former, equation $\left(24^{\prime}\right)$, provides the scaling of the temperature pedestal width.

\section{Sub-neoclassical heat transport}

It is interesting to compare the heat flux obtained from power balance at the plasma edge with the prediction of subneoclassical theory $[6,7]$ in the high collisionality regime:

$Q_{\mathrm{i}, \mathrm{s}-\mathrm{n}}=-2 \pi^{2} R(1+\kappa) a\left[1+1.6 q^{2}\left(1+\frac{Q^{2}}{S^{2}}\right)^{-1}\right] X_{\perp, \mathrm{i}} \partial_{r} T_{\mathrm{i}}$

where $X_{\perp, \mathrm{i}}=2 N_{\mathrm{i}} T_{\mathrm{i}} v_{\mathrm{i}} / m_{\mathrm{i}} \Omega_{\mathrm{i}}^{2}$ is the classical perpendicular heat conduction coefficient [22]. Introducing in (27) the values of the different parameters at the inflexion point of the temperature pedestal of the reference discharge [11] and noting that the sub-neoclassical factor

$1+\frac{Q^{2}}{S^{2}}=1+\alpha f_{\mathrm{inf}}^{2}+\beta f_{\mathrm{inf}}^{4}$

$=1+3.25 \times 0.31-2.49 \times 0.31^{2}=1.77$

(cf equation (14)) yields

$$
\begin{aligned}
2 \pi^{2} & \times 0.67 \times 2.62 \times 0.208\left[1+1.6 \times 3.4^{2} / 1.77\right] \\
& \times 2\left(1.87 \times 10^{20}\right)\left(0.69 \times 10^{5}\right) \\
& \times\left(0.35^{2} \times 10^{-6}\right)\left(1 / \sqrt{1.62} \times 0.6 \times 10^{-2}\right) \\
& \times\left(1.65 \times 10^{2}\right)\left(1.6 \times 10^{-19}\right)=0.90 \mathrm{MW} .
\end{aligned}
$$

The sub-neoclassical energy flux thus accounts for about half of the Ohmic input power $\left(P_{\mathrm{OH}}=1.84 \mathrm{MW}\right.$; the energy confinement time is $57 \mathrm{~ms}$ and the stored energy $105 \mathrm{~kJ}$ ), which 
confirms the important role of neoclassical transport in the edge pedestal. The second half of the input power is partly radiated $\left(P_{\mathrm{Rad}} \cong 0.5 \mathrm{MW}\right)$ and partly transported via the electron channel. To the extent that the above estimate $Q_{\mathrm{i}, \mathrm{s} \text {-n }}=$ $0.90 \mathrm{MW}$ is a conservative one, the neoclassical ion heat flux would be very close to, or even above, the difference between input and radiated power, if it were not for the sub-neoclassical reduction factor (1.77)! The high spatial resolution $(1 \mathrm{~mm}$ for the electron temperature profile, respectively $1.5 \mathrm{~mm}$ for the density profile) gives further confidence that the sub-neoclassical factor predicted by theory represents well the reality, although the ion temperature is actually needed.

A point of concern in neoclassical transport theory is that the heat flux is proportional to $N_{\mathrm{i}}^{2} Z_{\mathrm{eff}, \mathrm{i}} T_{\mathrm{i}}^{1 / 2} / L_{T}$, which typically decreases towards the edge. This behaviour may be balanced by an increase of the anomalous electron heat flux, but also by a decrease of the sub-neoclassical reduction factor which, as discussed earlier, has a maximum for $f \equiv$ $\Lambda / \Lambda_{\mathrm{s}}=f_{\mathrm{M}}$ (equation $(15 c)$ ). The precise values of the collisionality parameter at the boundaries between the various regimes is another question of concern; on the mere ground of power balance, we can safely conclude that the pedestal plasma must here be in the high collisionality regime, in agreement with the limits given in [23], since the theoretical expressions of the transport rate at intermediate and low collisionalities would lead to larger values of the fluxes (for identical plasma parameters).

\section{Summary of results, discussion and suggestions for further work (origin of ELMs)}

\subsection{Summary of results}

7.1.1. Plasma rotation and electric field. We have shown that the neoclassical theory of plasma rotation and electric field presented earlier [12] explains well the large ( $\cong 35 \mathrm{~km} \mathrm{~s}^{-1}$ ) co-current toroidal velocity measured in the core of Alcator C-Mod ELM-free high-confinement discharges [11] (cf section 5.1 and, in particular, figures 2 and 3). The characteristic electric field profiles reported by the DIII-D group [13,3] are also recovered. Further, the velocity reduction observed in discharges with an ITB and concomitant impurity accumulation [15] can be explained by an appropriate extension of the theory, which is presented here (section 5.2); the toroidal spin up and spin down observed during $\mathrm{L}-\mathrm{H}$ and $\mathrm{H}-\mathrm{L}$ transitions or ELM events can be explained similarly (section 5.3). It is worth noting that the necessity of that extension in the presence of enhanced radial main ion flows (in order to balance the impurity influx, or due to anomalous losses) had already been recognized in [12].

Under stationary conditions, the toroidal rotation velocity at a position $r$ within the plasma is proportional to the integral from the last closed magnetic surface up to $r$ of the product $U_{\theta, \mathrm{i}} \partial_{r} \ln T_{\mathrm{i}}$ (section 3). Toroidal rotation is therefore a global (rather than a local) property. In the H-mode, the rotation velocity of the core is primarily determined by the edge temperature pedestal where both $U_{\theta, \mathrm{i}}$ and $\partial_{r} \ln T_{\mathrm{i}}$ are largest; in Alcator C-Mod, the core toroidal rotation is in the co-current direction because the edge pedestal is in the high collisionality regime.
The situation is quite different in low-confinement discharges: here, the temperature and density gradients are weaker at the edge, so that comparable contributions to the above mentioned integral may arise from both core and edge; the core toroidal rotation velocity will clearly be smaller in absolute value; it may, moreover, be in the counter-current direction, as often observed, since the core is usually in the low colllisionality regime. The very relevance of the neoclassical results of [12] needs to be questioned here and other effects, such as those considered by Kim et al [31] and by Shaing [32], may become competitive.

7.1.2. Scaling laws. The requirements to validate scaling laws of the poloidal and toroidal rotation velocities as products of powers of the local plasma parameters or of the control parameters and the implications of such scaling laws have been discussed and compared to some experimental data [16] (section 5.3).

7.1.3. Power balance. We have also shown that the subneoclassical energy flux through the temperature pedestal calculated on the assumption of high collisionality [6,7] accounts for at least half the input power, leaving little room for anomalous ion transport. The sub-neoclassical factor being $\cong 1.77$, the standard neoclassical flux would account for almost the full input power! (section 6). As the neoclassical expressions for the other regimes would lead to higher energy fluxes, this result shows without any doubt that the pedestal plasma must be in the high collisionality regime.

\subsection{Discussion of the results}

The range of applicability of the theory is limited by various assumptions that we now review; examples of discharges where those are not satisfied are also briefly considered.

\subsubsection{The region which determines the core toroidal velocity} is in the high collisionality regime. In Ohmic PLT [33] and PDX [34] discharges, in JIPP-TIIU discharges heated by perpendicular neutral beam injection [35] and in JFT-2M discharges with balanced injection [36], the central toroidal velocity was measured to be in the counter direction with respect to the plasma current. The observations made in [33] have been justified earlier [12]. The results reported in [34-36] can be explained similarly. First of all, in the second case, $N_{\mathrm{e}} \approx 2.8 \times 10^{19} \mathrm{~m}^{-3}$ and $T_{\mathrm{i}} \approx 220 \mathrm{eV}$ at the plasma edge ( $R=1.11 \mathrm{~m}$; see figure 1$)$, which yields $0.045 q Z_{\text {eff }, \mathrm{i}}$ for the collisionality parameter defined in equation (3); that value is approximately a factor 10 lower than that estimated for Alcator C-Mod, justifying the direction reversal of the core toroidal rotation velocity; these conditions in JIPP-TIIU [35] are actually similar to those in PLT [33]. In the first case, the line-average density is $\approx 2.5 \times 10^{19} \mathrm{~m}^{-3}$ and, in the third, $N_{\mathrm{e}}(0) \approx(3.5-4.4) \times 10^{19} \mathrm{~m}^{-3}$; these values are again an order of magnitude lower than those in Alcator C-Mod and too small for the plasma to be in the high collisionality regime. Since the poloidal velocity changes sign at low collisionalities [14], the toroidal velocity predicted by equation $\left(8^{\prime}\right)$ must indeed be in the counter direction if, as mentioned in section 1 , the relation between toroidal 
and poloidal velocities obtained in the framework of the high collisionality neoclassical theory remains approximately valid in the other neoclassical collisionality regimes or in the presence of turbulence.

It is interesting to note, in this respect, that analysis of the JFT-2M discharges [36] has unveiled the existence of a nondiffusive term in the transport matrix [37]; the latter results in a spontaneous source of toroidal momentum in the direction anti-parallel to the plasma current. Equation (8) is compatible with that finding in the limit $U_{r, \mathrm{i}} \rightarrow 0$, the first term on the right-hand side playing the role of the toroidal momentum source. If we write equation (8) in a form similar to the expression of the radial momentum flux given in the text of [36], we find that the ratio of the diagonal and off-diagonal coefficients for momentum transport $\mu_{\varphi}$ and $\mu_{\varphi / \Delta T}$ can be identified with

$$
\begin{aligned}
\frac{\mu_{\varphi / \Delta T}}{\mu_{\varphi}} & =-0.107 q^{2}\left(1+\frac{Q^{2}}{S^{2}}\right)^{-1} \frac{B_{\varphi} U_{\theta, \mathrm{i}}}{B_{\theta} c_{\mathrm{i}}} \frac{\left(\partial_{r} T_{\mathrm{i}}\right) \partial_{r}\left(m_{\mathrm{i}} N_{\mathrm{i}} U_{\varphi, \mathrm{i}}\right)}{\left(\partial_{r} U_{\varphi, \mathrm{i}}\right) \partial\left(m_{\mathrm{i}} N_{\mathrm{i}} T_{\mathrm{i}}\right)} \\
& \approx-0.107 q^{2} \frac{B_{\varphi} U_{\theta, \mathrm{i}}}{B_{\theta} c_{\mathrm{i}}}
\end{aligned}
$$

this is negative if $U_{\theta, \mathrm{i}}>0$ (high collisionality regime) and positive otherwise; in the pedestal of the Alcator C-Mod ELMfree $\mathrm{H}$-mode, it reaches a minimum value of about -0.7 .

7.2.2. The role of charge exchange neutrals is negligible. The loss of momentum via charge exchange neutrals has been neglected in this paper. The critical neutral density below which this is permissible can be estimated as follows. We multiply equation (4) (where we set $U_{r, \mathrm{i}} \equiv \partial_{t} \equiv v_{\mathrm{i}, z} \equiv 0$ ) by $U_{\varphi, \mathrm{i}}$ and integrate over the minor radius of the discharge. Since $U_{\varphi, \mathrm{i}}\left(r_{\mathrm{s}}\right)=0$ and $\left.\partial_{r} U_{\varphi, \mathrm{i}}\right|_{r=0}=\left.\partial_{r} T_{\mathrm{i}}\right|_{r=0}=0$, we obtain

$$
\begin{aligned}
\int_{0}^{r_{\mathrm{s}}}[ & \left.m_{\mathrm{i}} v_{c x} N_{\mathrm{i}} U_{\varphi, \mathrm{i}}^{2}+\eta_{2, \mathrm{i}}\left(\partial_{r} U_{\varphi, \mathrm{i}}\right)^{2}\right] \mathrm{d} r \\
= & \int_{0}^{r_{\mathrm{s}}} \eta_{2, \mathrm{i}}\left(\partial_{r} U_{\varphi, \mathrm{i}}\right) 0.107 q^{2}\left(1+\frac{Q^{2}}{S^{2}}\right)^{-1} \\
& \times\left(\partial_{r} \ln T_{\mathrm{i}}\right)\left(\frac{B_{\varphi}}{B_{\theta}}\right) U_{\theta, \mathrm{i}} \mathrm{d} r .
\end{aligned}
$$

In view of the positive sign in the square bracket on the left-hand side, equation (29) shows that the toroidal rotation velocity gradient and, therefore, the toroidal velocity are reduced in the presence of neutrals; the role of these will be negligible only if

$$
\left(\frac{v_{c x}}{v_{\mathrm{i}}}\right)_{\mathrm{inf}} \leqslant \frac{1.2\left(a_{\mathrm{i}}^{2}\right)_{\mathrm{inf}}}{\kappa \Delta^{2}}
$$

(the charge exchange collision frequency is evaluated at the temperature pedestal inflexion point, as the toroidal rotation velocity is appreciable only inside that point). Introducing the characteristic parameters of the reference discharge [11] and $v_{c x} \cong \frac{1}{3}\langle\sigma v\rangle N_{0}$ (where $N_{0}$ is the neutral density, $\frac{1}{3}$ the approximate fraction of charge exchange neutrals leaving the plasma and $\langle\sigma v\rangle \cong 0.5 \times 10^{-13} \mathrm{~m}^{-3} \mathrm{~s}^{-1}$ the charge exchange rate) yields the condition

$$
\left(N_{0}\right)_{\text {inf }} \leqslant 1.05 \times 10^{16} \mathrm{~m}^{-3} \equiv 0.57 \times 10^{-4}\left(N_{\mathrm{i}}\right)_{\text {inf }} .
$$

The neutrals mean free path is approximately $0.68 \times 10^{-2} \mathrm{~m}$ if their temperature is $\left(T_{\mathrm{i}}\right)_{\mathrm{inf}} / 2$; this is comparable to the characteristic width $\kappa^{0.5} \Delta \cong 0.76 \times 10^{-2}$ m of the temperature pedestal. To the above critical neutral density at the inflexion radius thus corresponds a density of $\cong 2.85 \times 10^{16} \mathrm{~m}^{-3}$ at the LCFS.

It is worth noting that the heat flux carried to the walls by the charge exchange neutrals is only $5 \%$ of the sub-neoclassical heat flux $\left(27^{\prime}\right)$ when the neutral density reaches the critical value given in $(30 b)$.

7.2.3. The role of the geometry. The ellipticity of the cross section has been approximately taken into account by multiplying the length scales measured or mapped in the equatorial plane by the square root of the elongation. We show now that this approximation yields quantitatively correct estimates also for divertor geometry. Equations (4) and (5) are the result of large aspect ratio expansions of equations of the form

$$
\oint F(\Psi, \chi) \mathrm{d} \chi=0,
$$

where $\psi(\boldsymbol{r})$ is the flux function defined by $\nabla \psi=\boldsymbol{e}_{\psi} R B_{\chi}$ $\left(\boldsymbol{e}_{\psi}\right.$ is the unit vector perpendicular to the flux surface) and $\chi$ a generalized poloidal angle. In the kernel of (31), radial derivatives should be replaced by $R B_{\chi} \partial_{\psi}$ and the safety factor $q(r)$ by the local pitch angle of the magnetic field line $v(\Psi, \chi)=h_{\chi} B_{\varphi} / R B_{\chi}$, where $h_{\chi} \cong r$. The poloidal magnetic field $\mathrm{B}_{\chi}$ vanishes and the local pitch angle diverges at the location of the magnetic separatrix. As a consequence, the ratio $v^{2} /\left(1+Q^{2} / S^{2}\right)$, where $Q / S \propto \Lambda \propto h_{\psi}^{-1} v / B_{\chi} \propto v$ (see equation (6)), remains finite and the integrand in $\left(8^{\prime}\right)$ well behaved. Since the coils creating the separatrix are only acting over a short range, it becomes evident that our predictions will be barely affected by the divertor geometry.

\subsection{On the PVS K-H instability as a possible origin of the transition to EDA operation}

We divide both sides of equation (8) by $e B_{\theta} \sqrt{2} c_{\mathrm{i}} \partial_{r} \ln N_{\mathrm{i}}$ and obtain

$$
\frac{\partial_{r} U_{\varphi, \mathrm{i}}}{\sqrt{2} c_{\mathrm{i}} \partial_{r} \ln N_{\mathrm{i}}}=0.076 q^{2}\left(1+\frac{Q^{2}}{S^{2}}\right)^{-1} \eta_{\mathrm{i}}\left(\frac{B_{\varphi} U_{\theta, \mathrm{i}}}{B_{\theta} c_{\mathrm{i}}}\right) .
$$

(We again consider stationary discharges and neglect effects related to neutrals.) If the parameters at the pedestal inflexion point of the reference discharge [11] are introduced, the numerical value on the right-hand side of equation (32) is $0.98 / 1.77=0.55$ (see section 6 ), leading to the conclusion that this edge plasma is only barely stable according to the ideal K-H PVS instability threshold criterion [17]. In the fluid approximation, the ratio of the parallel and perpendicular mode numbers of the wave which becomes firstly unstable is (assuming $T_{\mathrm{e}} / T_{\mathrm{i}}=1$ )

$$
\frac{k_{z}}{k_{y}}=-\frac{a_{\mathrm{i}}}{\sqrt{2} L_{N}}
$$

whereas the linear growth rate maximizes for

$$
k_{y} a_{\mathrm{i}} \approx 0.5 \text {. }
$$


In Alcator C-Mod, the H-mode plasma frequently shows the characteristics referred to as EDA operation $[38,39,18]$ : in this state, there is still a pronounced pedestal, but the edge particle and impurity fluxes are increased relative to the ELMfree $\mathrm{H}$-mode. The apparent mechanism giving rise to EDA is a narrow band quasi-coherent oscillation; its frequency generally settles around $120 \mathrm{kHz}$ and its poloidal wave number is about $400 \mathrm{~m}^{-1}$. With the ion Larmor radius being of the order of $0.25 \times 10^{-3} \mathrm{~m}$ at the inflexion point of the temperature pedestal of the reference discharge, we obtain $k_{\theta} a_{\mathrm{i}} \approx 0.1$; this figure may be reconciled with the prediction of the twofluid theory [17] of the $\mathrm{K}-\mathrm{H}$ instability by noting that Landau damping shifts the short wavelength onset to smaller values of $k_{\theta}$ (and decreases the maximum growth rate) [40]. (It is noted here that the PVS K-H instability is not stabilized by magnetic shear unless the shear length $L_{\mathrm{s}}=q R / \hat{s}$ is comparable to or smaller than the density length scale $L_{N}$ [41], a condition not satisfied in tokamaks, and particularly not at the edge.) In the plasma frame of reference, the frequency is essentially the ion diamagnetic frequency: $f=\omega / 2 \pi=(2 \pi)^{-1} k_{\theta} T_{\mathrm{i}} / e B_{\varphi} L_{N}$; introducing $T_{\mathrm{i}} \approx 100 \mathrm{eV}$ (the temperature is slightly smaller in the EDA than in ELM-free $\mathrm{H}$-mode), $B_{\varphi} \approx 5 \mathrm{~T}$ and $L_{N} \approx 10^{-2} \mathrm{~m}$ yields $f \approx 127 \mathrm{kHz}$, in good agreement with the experimental value if $k_{\theta} a_{\mathrm{i}}=0.1$; the corresponding propagation velocity $\omega / k_{\theta} \approx 2 \mathrm{~km} \mathrm{~s}^{-1}$ also agrees with the measured value of $1.5 \mathrm{~km} \mathrm{~s}^{-1}$. Experimentally, the edge safety factor is the critical variable which determines which regime a discharge will be in: when $q_{\mathrm{s}}>4.0$ for standard shaped plasmas, the discharge is almost always EDA, while when $q_{\mathrm{s}}<3.5$, the plasma is almost always ELM-free [38,39]. These experimental results fit perfectly with equation (32), which shows that the ratio $\partial_{r} U_{\varphi, \mathrm{i}} / \sqrt{2} c_{\mathrm{i}} \partial_{r} \ln N_{\mathrm{i}}$ relevant to PVS $\mathrm{K}-\mathrm{H}$ instability onset increases rapidly with $q$, and with our above estimate for the ELM-free discharge with $q_{\mathrm{s}}=3.4$. In view of the pedestal sharp gradients, a careful study of the interplay between the PVS K-H instability and the EDA operation must take the non-local character of the mode into account.

\section{References}

[1] Wagner F. et al 1982 Phys. Rev. Lett. 491408

[2] Shaing K.C. and Crume E.C. 1989 Phys. Rev. Lett. 632369

[3] Burrell K.H. et al 1995 Proc. 15th Int. Conf. on Plasma Physics and Controlled Nuclear Fusion Research 1994 (Seville, 1994) vol 1 (Vienna: IAEA) p 221
[4] Diamond P.H. et al 1995 Proc. 15th Int. Conf. on Plasma Physics and Controlled Nuclear Fusion Research 1994 (Seville, 1994) vol 3 (Vienna: IAEA) p 323

[5] Moyer R.A. et al 1995 Phys. Plasmas 22397

[6] Rogister A.L. 1998 Phys. Rev. Lett. 813663

[7] Rogister A.L. 1999 Phys. Plasmas 6200

[8] Eriksson L.-G. et al 1997 Plasma Phys. Control. Fusion 3927

[9] Rice J.E. et al 1999 Nucl. Fusion 391175

[10] Chang C.S. et al 1999 Phys. Plasmas 61969

[11] Rice J.E. et al 2000 Phys. Plasmas 71825

[12] Claassen H.A., Gerhauser H., Rogister A. and Yarim C. 2000 Phys. Plasmas 73699

[13] Burrell K.H. et al 1992 Plasma Phys. Control. Fusion 341859

[14] Hazeltine R.D. 1974 Phys. Fluids 17961

[15] Rice J.E. et al 2001 Nucl. Fusion 41277

[16] Hughes J.W. et al 2002 Phys. Plasmas 93019

[17] D’ Angelo N. 1965 Phys. Fluids 81748

[18] Hutchinson I.H. 2001 Nucl. Fusion 411391

[19] Rogister A.L. et al 2001 Proc. 28th Eur. Conf. on Controlled Fusion and Plasma Physics (Funchal, 2001) (Geneva: European Physical Society) http://www.cfn.ist.utl.pt/EPS2001, paper P3.034

[20] Hutchinson I.H. et al 2000 Phys. Rev. Lett. 843330

[21] Rice J.E. et al 1997 Nucl. Fusion 37421

[22] Braginskii S.I. 1965 Reviews of Plasma Physics vol 1 (New York: Consultants Bureau) p 205ff

[23] Balescu R. 1988 Transport Processes in Plasmas vol 2 (Amsterdam: North-Holland) p 659

[24] Hinton F.L. and Wong K. 1985 Phys. Fluids 283082

[25] Connor J.W., Cowley S.C., Hastie R.J. and Pan L.R. 1987 Plasma Phys. Control. Fusion 29919

[26] Hogan J.T. 1984 Phys. Fluids 272308

[27] Stacey W.M. 1993 Phys. Fluids B 51413

[28] Stacey W.M. and Sigmar D.J. 1985 Phys. Fluids 282800

[29] Hinton F.L. and Hazeltine R.D. 1976 Rev. Mod. Phys. 48239

[30] Gangadhara S. et al 2001 J. Nucl. Mater. 598290

[31] Kim Y.B., Diamond P.H. and Groebner R.J. 1991 Phys. Fluids B 32050

[32] Shaing K.C. 2001 Phys. Rev. Lett. 86640

[33] Suckewer S. et al 1981 Nucl. Fusion 211301

[34] Brau K. et al 1983 Nucl. Fusion 231643

[35] Ida K. et al 1991 Nucl. Fusion 31943

[36] Ida K. et al 1995 Phys. Rev. Lett. 741990

[37] Itoh S.-I. 1992 Phys. Fluids B 4796

[38] Greenwald M. et al 2000 Plasma Phys. Control. Fusion 42 A263

[39] Hutchinson I.H. et al 1997 Proc. 16th Int. Conf. on Fusion Energy 1996 (Montreal, 1996) vol 1 (Vienna: IAEA) p 155

[40] Smith C.G. and von Goeler S. 1968 Phys. Fluids 112665

[41] Catto P.J., Rosenbluth M.N. and Liu C.S. 1973 Phys. Fluids 10 1719 